\title{
PRODUÇÃO CIENTÍFICA DE INOVAÇÃO NO BRASIL: UMÁANÁLISE SOB A ÓTICA DAS REDES SOCIAIS
}

Data de submissão: 22/06/2014

Aceite: 10/06/2016

Márcia Regina Santiago Scarpin ${ }^{1}$ Denise Del Prá Netto Machado ${ }^{2}$ Vanessa Edy Dagnoni Mondini ${ }^{3}$

Giancarlo Gomes ${ }^{4}$

\section{RESUMO}

A utilização das redes sociais na análise da produção científica permite a observação de aspectos interdisciplinares decorrentes da colaboração dos pesquisadores, além de proporcionar a análise e estruturação de um dado campo do conhecimento. Este estudo insere-se no âmbito das pesquisas que visam compreender as redes sociais no campo da produção científica na área de inovação, pretendendo quantificar a troca de informações para a construção do conhecimento. O estudo tem como objetivo identificar a formação de redes sociais na produção científica de inovação no período de 2006 a 2012, nos periódicos Qualis Capes de alto impacto (A1, A2, B1 e B2) do Brasil. A pesquisa é considerada descritiva, com método quantitativo e de caráter documental. Foram encontrados 678 autores, que representam os "nós" que, juntos, produziram 1.607 relações entre si (chamadas de "laços") de 459.006 laços possíveis, indicando uma rede social formada por laços fracos, fragmentada, com a existência de 133 subgrupos e de baixa densidade. Os autores intermediários, considerados os mais importantes em uma rede social, pois por meio deles um ator interage entre atores não adjacentes, compuseram-se pelos pesquisadores VACCARO G. L. R. e BITENCOURT M. P. Juntos, esses autores conectam uma rede formada por outros nove pesquisadores, a maior possível.

Palavras-chave: Redes; Inovação; Produção científica.

\footnotetext{
1 Possui graduação em Administração de Empresas pela Faculdade Estadual de Ciências Econômicas de Apucarana, UNESPAR emestrado em Administração pela Fundação Universidade Regional de Blumenau, FURB e doutorado em administração pela Fundação Getúlio Vargas - SP, FGV. Sao Paulo, SP - Brasil. E-mail:mrs.scarpin@gmail.com

2 Possui graduação em administração pela Universidade Federal de Santa Catarina, UFSC, mestrado em administração pela Universidade Federal de Santa Catarina, UFSC e doutorado em administração de empresas pela Fundação Getúlio Vargas - SP, FGV-SP. Atualmente é professora Titular do Programa de Pós-Graduação em Administração da Universidade do Sul de Santa Catarina - PPGA/UNISU. Florianópolis, SC-Brasil. E-mail: delpra@furb.br 3 Possui graduação em Comunicação Social Publicidade e Propaganda pela Fundação Universidade Regional de Blumenau, FURB, graduação em Gestão Comercial pela UNIASSELVI Centro Universitário Leonardo Da Vinci, UNIASSELVI, graduação em Tecnologia em Processos Gerenciais pela UNIASSELVI Centro Universitário Leonardo Da Vinci, UNIASSELVI, graduação em Administração pela UNIASSELVI Centro Universitário Leonardo Da Vinci, UNIASSELVI, mestrado em administração pela Fundação Universidade Regional de Blumenau, FURB e doutorado em Ciências Contábeis e Administração pela Fundação Universidade Regional de Blumenau, FURB. Gaspar, SC - Brasil. E-mail: prof.vanessa@ymail.com

4 Possui graduação em Administração pela Universidade do Oeste de Santa Catarina, UNOESC, mestrado em Administração pela Fundação Universidade Regional de Blumenau, FURB e doutorado em Ciências Contábeis e Administração pela Fundação Universidade Regional de Blumenau, FURB. E-mail: giancarlo@pzo.com.br
} 


\section{INTRODUÇÃO}

A inovação faz parte de um acúmulo constante de conhecimentos e capacitação tecnológica contínua. Sua importância já foi evidenciada nos estudos de Schumpeter (1911), que introduziu o conceito de inovação na análise econômica e a retratou como grande mola propulsora para o desenvolvimento. As redes sociais impactam tanto a difusão de inovações quanto a propagação da informação e do conhecimento que oportuniza o desenvolvimento de inovações (WATTS; STROGATZ, 1998; SCOTT, 2000; OTTE; ROUSSEAU, 2002; HANNEMAN; RIDDLE, 2005; LIU et al., 2005).

Pesquisas que analisam a produção científica sobre inovação no âmbito acadêmico têm surgido visando verificar como está a qualidade e a produção científica dessa área. No âmbito nacional, pode-se citar os trabalhos de Bignetti (2006), Bignetti, Cappra e Thomas (2008), Muylder et al. (2008), Machado, Gomes e Giotto (2008), Gomes, Machado e Giotto (2009), Ropelato, Silveira e Machado (2010), Kneipp et al. (2011) e De Andrade Júnior e Ceranto (2013). Já entre os autores que empregaram a análise de redes sociais em seus estudos, o destaque vai para os trabalhos de Wasserman e Faust (1994), Scott (2000), Bulgacov e Verdu (2001), Newman (2001a), Moody (2004), Barabási (2005), Guimerà et al. (2005), Liu et al. (2005), Larivière, Gingras e Archambault (2006), Rossoni, Hocayen-da-Silva e Ferreira Júnior (2008).

Este estudo insere-se no âmbito das pesquisas que objetivam compreender as redes sociais no campo da produção científica, mais especificamente na área de inovação, pretendendo quantificar a troca de informações e a construção do conhecimento. O conhecimento científico é construído socialmente, sendo influenciado pelos pares que compõem estruturalmente a rede de relações entre as instituições, objetivando não apenas descrever tais relacionamentos, mas também entender como tal estrutura afeta a produção do conhecimento (ROSSONI; HOCAYENDA-SILVA; FERREIRA JR., 2008).

Dessa forma, emerge a questão de pesquisa que norteia este estudo: quais são as redes sociais formadas a partir da produção científica sobre inovação dos periódicos de alto impacto, brasileiros, listados na Coordenação de Aperfeiçoamento de Pessoal de Nível Superior (Capes), classificados como Qualis A1, A2, B1 e B2? Para responder a essa questão de pesquisa, o estudo tem como objetivo identificar a formação de redes sociais na produção científica de inovação no período 2006-2012, nos periódicos Qualis Capes de alto impacto (A1, A2, B1 e B2) do Brasil.

Para alcançar o objetivo proposto, buscou-se levantar os artigos publicados nos periódicos considerados de alto impacto entre os anos de 2006 e 2012; identificar quais as universidades que atuam nesta rede; identificar o tipo de pesquisa utilizado na metodologia dos artigos relacionados; e analisar a formação das redes sociais nesses periódicos.

Para tanto, este estudo encontra-se estruturado com mais quatro seções, além desta primeira, que é a introdução. Na segunda, como referencial teórico, destacam-se a teoria das redes (MILGRAM, 1967; KNOKE; KUKLINSKI, 1982; BURT, 1992; NOHRIA, 1992; WASSERMAN; FAUST, 1994; UZZI, 1997; WATTS, STROGATZ, 1998; SCOTT, 2000; GNYAWALI, MADHAVAN, 2001; HANNEMAN; RIDDLE, 2005; LIU et al., 2005) e inovação (SCHUMPETER, 1911, 1982; DOSI, 1982; VAN DE VEN; POOLE, 1990; VAN DE VEN; ANGLE, 1989, 1999; DAMANPOUR, 1991; ROTHWELL, 1994; AFUAH, 2003; TIDD; BESSANT; PAVITT, 2005; HAMEL, 2006). A terceira é a seção destinada à metodologia da pesquisa. Na quarta, apresenta-se a análise dos dados. Por fim, a quinta seção destina-se às considerações finais e a sugestões de futuras pesquisas. 


\section{REFERENCIAL TEÓRICO}

Nesta etapa, serão apresentadas as teorias de redes e inovação.

\subsection{Teoria de redes}

O estudo de redes pode abranger desde a ciência da computação até a sociologia. As redes sociais criam conexões entre pessoas no seu cotidiano (RAPOPORT; HORVATH, 1961), contatos profissionais, negócios empresariais (PODOLNY; PAGE, 1998; LAZZARINI, 2007), relações acadêmicas (PRICE, 1965; REDNER, 1998; GUIMARÃES et al., 2009; ROSSONI; GUARIDO FILHO, 2009; MARTINS, 2009).

A rede tanto fortalece as relações como contribui para o aperfeiçoamento, a maturação ou mesmo a inovação do conhecimento científico, que, conforme Kuhn (1978) e Popper (1972), é construído socialmente. As redes sociais influenciam diretamente a forma como os pesquisadores pensam e formulam suas ideias (LIU et al., 2005).

O presente estudo utiliza as redes sociais derivadas da sociologia e da antropologia com enfoque nas relações entre pesquisadores da área de inovação, podendo ser chamada de análise de redes sociais (ARS) ou Social Network Analysis (SNA). É definida como conjuntos de contatos que ligam vários atores, nos quais tais contatos podem ser de diferentes tipos e apresentar conteúdos distintos, bem como diversas propriedades estruturais (NELSON, 1984), ou ainda como um conjunto finito de atores e de relações que ocorrem entre eles (WASSERMAN; FAUST, 1994).

As redes "são sistemas compostos por 'nós' e conexões entre eles, que, nas ciências sociais, são representados por sujeitos sociais (indivíduos, grupos, organizações etc.) conectados por algum tipo de relação" (SILVA et al., 2006, p. 77). A análise de redes sociais é baseada na premissa de que as relações entre atores sociais podem ser descritas por um gráfico (Liu et al., 2005). A formação das redes sociais constitui-se de: ator, nós, laço relacional (ligações fortes ou fracas), díade, tríade, grupo e centralidade (WASSERMAN; FAUST, 1994; LIU et al., 2005).

Os atores representam os "nós" (HANNEMAN; RIDDLE, 2005). A ligação de um ou mais tipos específicos de interdependência é considerada os laços (BERKOWITZ, 1982). Esses laços podem caracterizar-se como: laços fortes, que correspondem a uma rede social formada por uma densa malha de relacionamentos, ou laços fracos, que são aqueles feitos fora de um círculo coeso de relacionamento (WASSERMAN; FAUST, 1994).

A ligação entre dois atores numa unidade de análise é considerada uma díade, e as tríades estão presentes nas ligações entre três ou mais atores. Os grupos têm sua origem nas relações que possuem laços mensuráveis, caracterizando-se por um conjunto finito de atores definidos por critérios conceituais, teóricos ou empíricos. Já a centralidade consiste em identificar os atores mais importantes em uma rede social. Quanto mais centrais são esses atores na rede social, mais se evidencia sua importância (WASSERMAN; FAUST, 1994).

Um dos tipos de rede que possuem alto grau de agrupamento e baixa distância média entre os nós é a Small World ou "Mundo Pequeno" (MILGRAM, 1967; WATTS; STROGATZ, 1998). Essa rede remete à situação em que um indivíduo pode acessar qualquer outro a partir de seus relacionamentos: mesmo que a maioria das pessoas não esteja relacionada diretamente entre si, ela se conecta indiretamente por meio de poucos intermediários (LAZZARINI, 2007). Watts e Strogatz (1998) sugerem que esse fenômeno ocorre quando atores em uma esparsa rede estão altamente agrupados, mas, ao mesmo tempo, conectados a atores fora de seus grupos, por meio de um pequeno número de intermediários. 
Várias são as maneiras de identificar a estrutura e as relações de uma rede, entre as quais se destacam: propriedades estruturais, com medidas de densidade e centralidade; papéis e posições, com análise de clusters; e análise estatística dos relacionamentos, com testes de proposições teóricas acerca das propriedades relacionais (WASSERMAN; FAUST, 1994).

No caso das propriedades estruturais, a densidade evidencia a proporção do número de laços efetivamente realizados em uma rede em relação ao número total de possibilidades entre os atores da rede (KNOKE; KUKLINSKI, 1982; SCOTT, 2000). Gnyawali e Madhavan (2001) destacam três características das redes densas: a) facilitam o fluxo de recursos, principalmente a informação; b) funcionam como sistemas fechados de confiança, gerando mais facilmente comportamentos similares; e c) facilitam a atribuição de sanções.

A centralidade, por sua vez, configura-se como uma propriedade dos atores, que mede o quanto eles são centrais em uma rede. Ela permite avaliar o grau com que um ator específico é capaz de, direta ou indiretamente, acessar outros atores na rede, podendo ser caracterizada como: a) centralidade de grau (degree), que consiste no número de laços que um ator possui com outros em uma rede (como ela leva em conta somente os relacionamentos adjacentes, tal medida revela somente a centralidade local dos atores); b) centralidade de proximidade, que é baseada na proximidade ou distância de um ator em relação aos outros em uma rede (a medida de centralidade de proximidade [closeness] de um ator é obtida por meio da soma das distâncias geodésicas entre todos os outros atores); e c) centralidade de intermediação (betweenness), na qual a interação entre atores não adjacentes pode depender de outros atores, que podem potencialmente ter algum controle sobre as interações entre dois atores não adjacentes. Sendo assim, um ator é um intermediário que se liga a vários outros que não se conectam diretamente (SCOTT, 2000; WASSERMAN; FAUST, 1994).

A relação entre atores ou grupos pode ser chamada de clusters, cliques, clans, componentes, cores ou ainda ciclos e pode ser identificada quando os laços entre os atores de um grupo identificam subgrupos coesos que podem ser formalizados expondo as suas muitas diferentes propriedades. Esses subgrupos podem ser pensados como um conjunto de atores no qual todos escolhem a todos como pares em suas ligações, formadas por três ou mais atores da rede (SCOTT, 2000; WASSERMAN; FAUST, 1994).

Os subgrupos podem ser baseados na alcançabilidade e na proximidade de indivíduos e são formalizados pelo conceito $n$-clique, que é considerado a distância geodésica entre os pontos. Os subgrupos coesos requerem uma pequena distância entre os nós, na qual a maior distância geodésica entre dois nós é menor ou igual a $n$, sendo $n$ o caminho máximo em que membros de um clique podem estar conectados (WASSERMAN; FAUST, 1994; SCOTT, 2000).

Por fim, os métodos de análise estatísticos possibilitam avaliar as redes tanto em nível local (díades e tríades) quanto em nível global. Três são as finalidades para utilização de ferramentas estatísticas defendidas por Hanneman e Riddle (2005): 1) comparar duas relações no mesmo conjunto de atores; 2 ) explicar o impacto de atributos nos relacionamentos e vice-versa; e 3) explicar as relações entre os atores na rede.

A partir de um estudo pioneiro, realizado por Newman (2001b), no campo da produção científica, o autor identificou que autores que entram na rede tendem a entrar preferencialmente a partir dos autores mais conectados. Alguns estudos recentes sobre redes sociais, como o de Martins (2009), demonstraram que, no campo de gestão de operações, a grande maioria das publicações possuiu estrutura de coautoria, sendo que cresceu o número médio de laços de coautoria entre estes pesquisadores, o que indicou que os autores tenderam a relacionar-se mais agora do que no passado, porém o volume de laços efetivamente construídos entre os autores não acompanhou o aumento de laços possíveis na rede, o que levou a uma maior fragmentação desta. 
Rossoni e Guarido Filho (2009) realizaram um levantamento de artigos publicados na área de administração e afins, dos cursos de pós-graduação em Administração, e constataram um componente principal coerente com a caracterização estrutural do tipo small worlds, em que agrupamentos de programas de pós-graduação se apresentavam como vizinhanças bem definidas, que, por sua vez, não estavam isoladas de outros grupos, mas interligadas por meio de poucos intermediários. Na mesma linha, Guimarães et al. (2009) investigaram a formação de redes sociais entre pesquisadores de programas de pós-graduação, evidenciando que a rede pesquisada é pouco densa, com relações esparsas e, na maioria das vezes, fracas entre programas.

Analisando áreas específicas, Martins et al. (2010) investigaram a rede de pesquisadores da área de gestão de operações no Brasil e verificaram a existência de uma rede fragmentada e pouco densa, além da presença de alguns grupos coesos e próximos que conferem estabilidade ao campo (redes small worlds). Ressaltaram ainda que o efeito dos laços não redundantes é moderado pela centralidade do pesquisador e o efeito sobre a produtividade é maior entre pesquisadores com maior grau de centralidade. Mendes-da-Silva, Onusic e Giglio (2013) estudaram as propriedades estruturais das redes de relações entre os pesquisadores na área de finanças no Brasil. Por meio de Análise de Redes Sociais, verificaram que: o ambiente brasileiro é caracterizado por small worlds, poucos pesquisadores conseguem manter a regularidade na produção e a centralidade maior dos pesquisadores na rede implica um volume maior de artigos por eles publicados.

\subsection{Redes de inovação}

O mundo vive a era da inovação (BART, 2004). A importância do tema, nos últimos anos, é evidenciada por muitos autores que vêm estudando o assunto, tornando seu conceito mais extenso e complexo (SCHUMPETER, 1911, 1982; DOSI, 1982; VAN DE VEN; ANGLE, 1989; DAMANPOUR, 1991; ROTHWELL, 1994; AFUAH, 2003; TIDD; BESSANT; PAVITT, 2005; HAMEL, 2006). Apesar dos numerosos estudos, não há consenso sobre sua definição (WAN; ONG; LEE, 2005). Segundo Monteiro, Sacomano Neto e Giuliani (2013), na literatura científica contemporânea, o termo "inovação" possui um sentido mais preciso do que o senso comum atribui a essa palavra. Para Barbieri (1997), dependendo da área de estudo, o termo "inovação" apresenta diferentes significados.

A inovação gera vantagem competitiva para as organizações, criando valor para aqueles que a utilizam, sendo um fator de diferenciação na elaboração de possíveis estratégias (SCHUMPETER, 1911, 1982). Ela pode ser entendida como a adoção de uma ideia já existente, mas que é nova para a organização que a está adotando, incluindo novos produtos, serviços, tecnologias, processos, procedimentos, sistemas ou arranjos sociais (DOSI, 1988; PENNINGS, 1998; AFUAH, 2003). Damanpour (1991) complementa essa noção incorporando ao contexto novos comportamentos, uma nova estrutura ou sistema administrativo e um novo plano ou programa relativo aos membros da organização, os quais, quando ligados à gestão, trabalham para transformar input em output (HAMEL, 2006).

Além de uma ideia, a inovação é uma prática ou um bem material que é percebido como novo e de relevante aplicação (ZALTMAN; DUNCAN; HOLBEK, 1973), podendo ser baseada no uso de um novo conhecimento tecnológico ou de mercado para oferecer produtos ou serviços novos aos clientes. Neste caso, considera-se um produto novo quando se consegue baixar seu custo, seus atributos são melhorados, ou ainda quando eles são inexistentes no mercado (AFUAH, 2003).

Os pesquisadores do Minnesota Innovation Research Program (MIRP), por meio dos estudos do Minnesota Innovation Survey (MIS), definiram a inovação como um processo de desenvolvimento e implantação de uma novidade, incluindo novos processos ou o desenvolvimento de novas ideias, como uma nova tecnologia, produto, processo organizacional ou novos arranjos (VAN DE VEN; ANGLE, 1989). 
Analisando as definições apresentadas, é possível congregá-las em um conceito como o utilizado pela empresa 3M, no qual a inovação representa uma equação algébrica: ideia + ação = resultado (GUNDLING, 1999). Independentemente dos conceitos apresentados, é possível observar que o processo de inovação não surge de forma isolada. É necessário que o ator se integre a uma rede para gerá-la e favorecê-la. Lemos, Lastres e Albagli (1999, p. 135) afirmam que a formação de redes é o "formato organizacional mais adequado para promover o aprendizado intensivo para a geração de conhecimento e inovações".

Com isso, além do ambiente interno, as organizações também podem se favorecer das inovações geradas pelas universidades e centros de pesquisa, embora essa relação seja mais forte com as grandes e pequenas empresas de bases tecnológicas, enquanto as demais se beneficiam com a apropriação de novos conhecimentos (ARBIX; NEGRI, 2009). Gardner (1999) entende a importância do meio acadêmico, principalmente das universidades e fundações, que contribuem como elo entre o governo e o setor privado.

A interação é essencial para a geração de inovação. Lemos, Lastres e Albagli (1999) afirmam que a organização não inova sozinha; ela precisa de fontes de informações e conhecimentos que podem estar dentro ou fora da organização. Por isso, a inovação é um processo interativo que ocorre com a contribuição de vários agentes (econômicos e sociais). Para Monteiro, Sacomano Neto e Giuliani (2013), um dos fatores para o desenvolvimento do potencial de inovação é a capacidade de uma organização estabelecer relacionamentos entre si em uma rede de organizações.

As redes sociais são recursos importantes para a inovação, em virtude de manterem canais e fluxos de informação em que a confiança e o respeito entre atores os aproximam e os levam ao compartilhamento de informações que incidem no conhecimento detido por eles, modificando-o ou ampliando-o (TOMAÉL; ALCARÁ; DI CHIARA, 2005).

De acordo com Silva et al. (2006), a utilização das redes sociais na análise da produção científica permite a observação de aspectos interdisciplinares decorrentes da colaboração dos pesquisadores, além de proporcionar a análise e estruturação de um dado campo do conhecimento, contribuindo para as duas correntes teóricas existentes: uma que considera os aspectos da inovação sob a ótica microeconômica da firma e outra que aborda o processo de inovação como um paradigma econômico, com um maior vínculo dos seus efeitos sobre o ambiente social e econômico (CONCEIÇÃO, 2000).

Pereira e Reinert (2013) afirmam que as redes sociais são fundamentais tanto na formação quanto na evolução da empresa, provendo recursos e informações que auxiliam na criação e no desenvolvimento da inovação. Participar de redes de inovação, segundo Monteiro, Sacomano Neto e Giuliani (2013), resulta em maior competitividade e capacidade organizacional de adaptação ao ambiente. Em função de serem construídas com o cliente, redes de inovação favorecem a fluidez das informações e, consequentemente, o ajuste das estratégias mercadológicas às necessidades do público-alvo, atendendo-os de forma mais específica.

\section{PROCEDIMENTOS METODOLÓGICOS}

Este estudo é uma pesquisa descritiva, com método quantitativo e de caráter documental. A pesquisa descritiva é feita por meio de observação, registro, análise e correlação dos fatos ou fenômenos sem manipulá-los (CERVO; BERVIAN, 2002). Esta pesquisa também tem abordagem quantitativa, na qual foram aplicadas as técnicas estatísticas de análise de redes no tratamento dos dados coletados. A pesquisa documental foi utilizada no estudo bibliométrico e sociométrico. Martins e Theóphilo (2007) destacam que a pesquisa documental realiza levan- 
tamento de material editado, como livros, periódicos, entre outros. Por sua vez, as pesquisas bibliométricas, conforme Macias-Chapula (1998, p. 134), compreendem "o estudo dos aspectos quantitativos da produção, disseminação e uso da informação registrada". No que concerne às pesquisas sociométricas, elas se voltam à exploração da matriz de relacionamentos oriunda dos atores sociais (GALASKIEWICZ; WASSERMAN, 1994).

A população da pesquisa compreendeu 116 periódicos de alto impacto, brasileiros, classificados pelo sistema Qualis da Capes. O Qualis é um conjunto de procedimentos utilizados para a estratificação da qualidade da produção intelectual dos programas de pós-graduação. Ele afere a qualidade dos artigos e de outros tipos de produção, a partir da análise da qualidade dos veículos de divulgação, ou seja, periódicos científicos e anais de eventos. A classificação de periódicos e eventos é realizada pelas áreas de avaliação e passa por processo anual de atualização. Esses veículos são enquadrados em estratos indicativos da qualidade - A1, o mais elevado; $A 2$; $B 1 ; B 2$; B3; B4; B5; C-com peso zero (CAPES, 2011).

Para fins da pesquisa, foram adotados os periódicos classificados como A1, A2, B1 e $\mathrm{B} 2$, brasileiros, da área de administração, contabilidade e turismo, por se entender que o tema "inovação" pode estar presente em diversas linhas de pesquisa, além da administração. Porém ressalta-se que se buscou identificar a palavra "inovação" voltada às organizações, de forma que foram excluídos artigos da área da saúde, na qual o tema era abordado em um contexto de descoberta clínica. Os demais periódicos, B3, B4, B5 e C, e os periódicos internacionais, por questões de delimitação deste estudo, não foram contemplados. Dos 116 periódicos levantados, 45 possuíam algum artigo relacionado ao tema "inovação", totalizando 378 artigos publicados.

A palavra "inovação" foi pesquisada no título, no resumo e nas palavras-chave. Entretanto, todos os resumos foram analisados no intuito de verificar se a palavra "inovação" estava inserida no contexto organizacional. O levantamento foi realizado no ano de 2013. Além dos autores, também foram levantadas as instituições às quais eles pertencem ou pertenciam na época da publicação do artigo, informação esta consultada no próprio artigo ou no Curriculum Lattes de cada autor. Na ocorrência de algum pesquisador trabalhar em duas universidades simultaneamente, foi considerada a primeira mencionada.

Também se buscou identificar a abordagem adotada nos artigos, sendo esta classificada como qualitativa e quantitativa, bem como as duas abordagens juntas, ou seja, qualiquantitativa.

Quanto aos procedimentos adotados na análise quantitativa dos dados, utilizou-se a estatística descritiva para o levantamento da produção científica sobre inovação, por meio dos periódicos classificados como A1, A2, B1 e B2, disponíveis na relação da Capes (2008). Na análise das redes sociais estabelecidas entre os autores e suas respectivas instituições, recorreu-se ao software UNICET $^{\circ}$ versão 6.0. De acordo com Hanneman e Riddle (2005), existem duas razões para a utilização de métodos estatísticos em análise de redes. A primeira, no caso de grandes redes, é a possibilidade de descrever e entender padrões de comportamento seja da rede como um todo, seja de seus atores imersos. A segunda razão é a possibilidade de entender o processo de evolução de redes no tempo, a partir da probabilidade de ações dos atores.

Todo método tem limitações. Segundo Vergara (2000, p. 59), "é saudável antecipar-se às críticas que o leitor poderá fazer ao trabalho, explicitando quais as limitações que o método escolhido oferece, mas que ainda assim o justificam como o mais adequado aos propósitos da investigação". O corte transversal, não sendo constituída uma série histórica e havendo o registro desatualizado de alguns periódicos, faz parte desse contexto. 


\section{APRESENTAÇÃO E ANÁLISE DOS DADOS}

Esta seção está estruturada de acordo com os objetivos do estudo e com as variáveis mensuradas. A finalidade deste estudo não foi levantar o número de artigos publicados por periódicos, e sim os autores que tiveram publicações sobre o tema "inovação" nos periódicos de alto impacto do sistema Qualis Capes (A1, A2, B1 e B2), para, assim, verificar qual a rede que se estabelece por meio deles. Porém, em consequência da abordagem de pesquisa utilizada pelos autores, também foi levantado o número de artigos publicados no período 2006-2012, conforme mostra a Tabela 1.

Tabela 1 - Abordagem adotada

\begin{tabular}{c|c|c}
\hline Periódico & Número de artigos & Porcentagem \\
\hline Qualitativa & 294 & $78 \%$ \\
\hline Quantitativa & 75 & $20 \%$ \\
\hline Quanti-Quali. & 09 & $2 \%$ \\
\hline TOTAL & 378 & $100 \%$ \\
\hline
\end{tabular}

Fonte: dados da pesquisa.

Com relação à abordagem adotada, verificou-se que os estudos qualitativos predominam, com um total de 294 artigos, representando $78 \%$ do total levantado. Os estudos quantitativos representaram $20 \%$ do total, com 75 publicações. Apenas $2 \%$ ou nove artigos foram classificados como quantitativos e qualitativos, configurando a rede de inovação que se forma por meio dos autores que desenvolvem essas pesquisas.

A inovação é um processo interativo que ocorre com a contribuição de vários agentes (econômicos e sociais). As redes sociais são recursos importantes para a inovação, pois aproximam os autores, levando-os a compartilhar informações que incidam no conhecimento detido por eles (TOMAÉL; ALCARÁ; DI CHIARA, 2005). Nesse contexto, a rede social de inovação, aqui representada na Figura 1, também chamada de análise de redes sociais (ARS) ou Social Network Analysis (SNA), na qual são apresentadas as relações entre os atores, bem como os componentes isolados da rede, é composta de 678 autores, denominados de "atores", que representam os "nós" (HANNEMAN; RIDDLE, 2005). Esses atores produziram 1.607 relações entre si. Tal interdependência é chamada de "laços" (BERKOWITZ, 1982).

Figura 1-Análise de rede social de inovação 




Fonte: dados da pesquisa.

A Figura 1 apresenta os laços entre os "nós" compostos pelos diversos atores que fazem parte dessa rede. Dos 678 autores pertencentes à rede de inovação, a maioria deles encontra-se conectada. As contribuições da produção em rede estão tanto no fortalecimento das relações como na contribuição para o aperfeiçoamento, a maturação ou mesmo a inovação do conhecimento científico, que são construídos socialmente (KUHN, 1978; POPPER, 1972).

Pela análise da rede social de inovação, mostrada na Figura 1, é possível perceber uma rede fragmentada. Sua densidade foi calculada em 0,0035 ou $0,35 \%$, indicando uma rede de baixa densidade. A densidade evidencia a proporção do número de laços efetivamente realizados em relação ao número total de possibilidades entre os atores da rede (KNOKE; KUKLINSKI, 1982; SCOTT, 2000). Neste estudo, foram levantados 1.607 laços realizados em 459.006 laços possíveis. Sendo assim, essa rede é formada por laços fracos, ou seja, laços feitos fora de um círculo coeso de relacionamento (WASSERMAN; FAUST, 1994).

Resultado parecido foi constatado no estudo desenvolvido por Guimarães et al. (2009), no qual as redes sociais entre pesquisadores de programas de pós-graduação evidenciaram-se pouco densas, com relações esparsas e, na maioria das vezes, fracas entre programas. Os autores destacam ainda que uma rede entre os programas de pós-graduação pode constituir-se condição ideal para aumentar o volume e a qualidade da produção científica brasileira. Da mesma forma, 
ao analisarem a rede de pesquisadores da área de gestão de operações no Brasil, Martins et al. (2010) verificaram a existência de uma rede fragmentada, pouco densa e composta por alguns grupos coesos e próximos que conferem estabilidade ao campo (redes small worlds).

Uma rede mais densa em princípio significa difusão mais rápida de informações, na qual o conhecimento percorre com maior agilidade e fluidez aqueles conectados a ela, contribuindo para a ampliação, a diversificação e as inovações do capital intelectual. Além disso, as redes densas facilitam o fluxo de recursos, especialmente de informação. Elas funcionam como sistemas fechados de confiança, gerando comportamentos similares, e promovem a atribuição de sanções (GNYAWALI; MADHAVAN, 2001).

Com o intuito de identificar a estrutura e as relações dessa rede social de inovação, foram analisadas as medidas de densidade, grau de centralidade, centralidade de proximidade, centralidade de intermediação e análise dos clusters. Outros indicadores podem ser utilizados para a análise de uma rede, porém por delimitação do estudo optou-se por esses descritos.

\subsection{Grau de centralidade (centrality degree)}

A centralidade de uma rede permite identificar a posição em que um ator se encontra em relação aos demais na rede (HANNEMAN; RIDDLE, 2005). Da mesma forma, ela permite avaliar o grau com que um ator específico é capaz de, direta ou indiretamente, acessar outros atores na rede. A Tabela 2 apresenta o grau de centralidade (centrality degree), que consiste no número de laços que um ator possui com outros atores em uma rede. Foram separados os dez atores com maior número de laços.

Tabela 2 - Grau de centralidade (centrality degree)

\begin{tabular}{c|c|c}
\hline Classificação & Principais atores & Degree \\
\hline 1 & GOMES, C. M. & 10.000 \\
\hline 2 & MACHADO, D. P. N. & 10.000 \\
\hline 3 & PORTO, G. S. & 10.000 \\
\hline 4 & PEREIRA, R. S. & 9.000 \\
\hline 5 & BINOTTO, E. & 9.000 \\
\hline 6 & VACCARO, G. L. R. & 7.000 \\
\hline 7 & SIQUEIRA, E. S. & 7.000 \\
\hline 8 & BITENCOURT, M. P. & 7.000 \\
\hline 9 & ESTEVES, G. & 7.000 \\
\hline 10 & KNEIPP, J. M. & 7.000 \\
\hline
\end{tabular}

Fonte: dados da pesquisa.

É possível observar, pela Tabela 2, que os atores GOMES C. M., MACHADO D. P. N., PORTO G. S., PEREIRA R. S., BINOTTO E., VACCARO G. L. R., SIQUEIRA E. S., BITENCOURT M. P., ESTEVES G. e KNEIPP J. M. são os autores que mais laços possuem. Porém, como são levados em conta somente os relacionamentos adjacentes, tal medida revela apenas a centralidade local dos atores (SCOTT, 2000), conforme apresentado na Figura 2. 


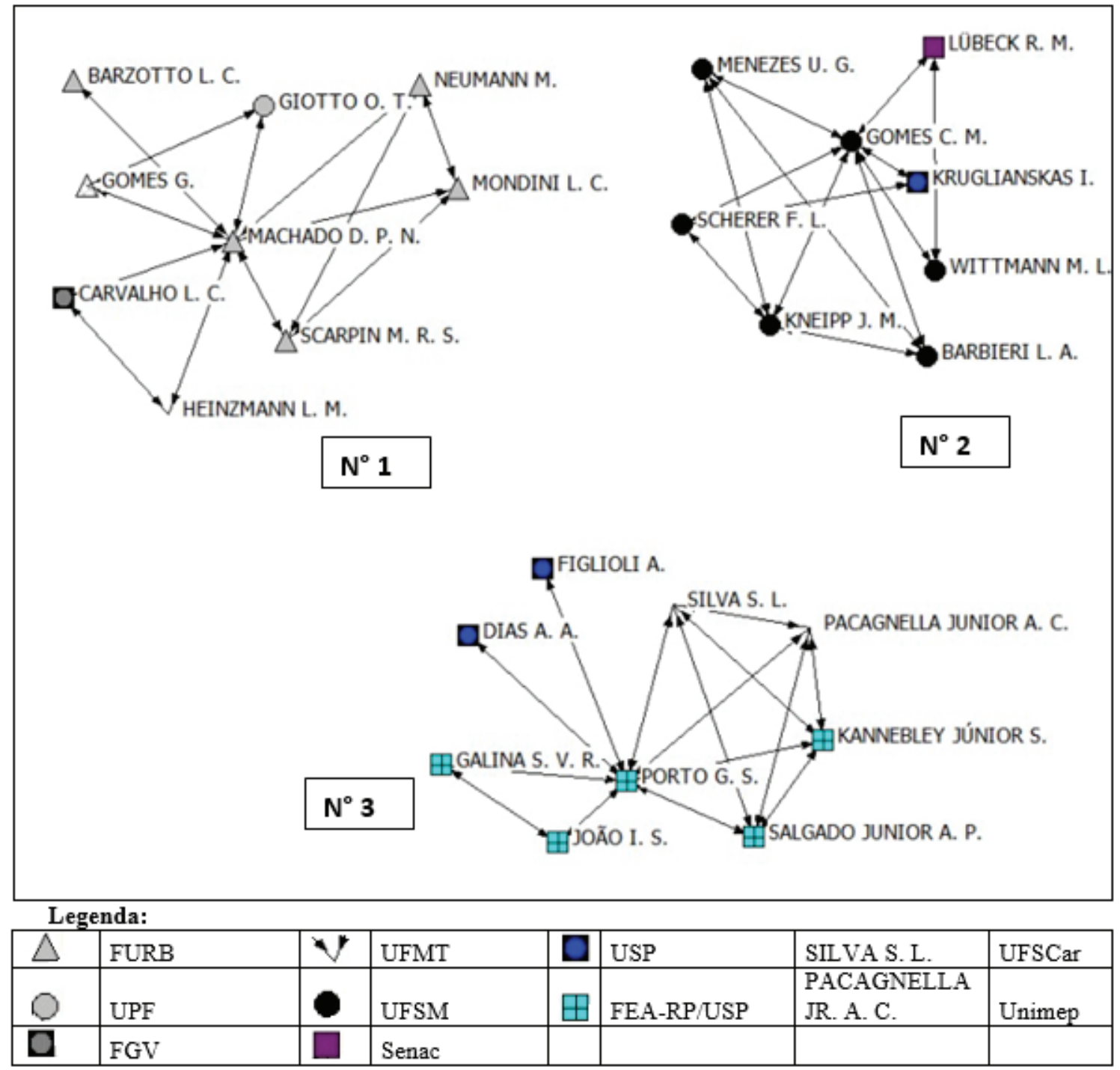

Fonte: dados da pesquisa.

Analisando a Figura 2, é possível observar que o número de laços produzidos por cada autor é um componente determinante para a análise de uma rede social, pois sem eles a formação da rede não ocorreria. Cada rede isolada (N.1, N.2 e N.3) representa um conjunto de atores que compartilham conhecimento e desenvolvem trabalhos em equipe, porém restritos ao seu grupo, limitando o conhecimento entre os seus pares, uma vez que, em sua maioria, pertencem à mesma universidade. Isso pode gerar uma homogeneidade, criando um obstáculo ao conhecimento heterogêneo na produção do conhecimento na área de inovação. A importância de um ator na rede não se dá somente pelo número de contatos diretos que ele mantém, mas também pelo número de contatos que intermedeia (ROSSONI; GUARIDO FILHO, 2009).

Martins et al. (2010) ressaltam que o efeito dos laços é moderado pela centralidade do pesquisador. $O$ efeito sobre a produtividade é maior entre pesquisadores com maior grau de centralidade. Já Mendes-da-Silva, Onusic e Giglio (2013) afirmam que o ambiente brasileiro tem como características a constituição por small worlds, a baixa regularidade de produção e o volume de produção maior de pesquisadores com maior grau de centralidade. 
É oportuno ressaltar que os laços não revelam os atores mais relevantes de uma rede, pois é possível haver um número expressivo de laços sem conexões com o restante da rede, indicando uma preferência de produção limitada a determinado conjunto de autores. Se há baixo acoplamento entre atores, a quantidade total de poder na rede também é baixa, pois há poucos contatos entre os atores para que esse poder possa ser exercido (HANNEMAN, RIDDLE, 2005).

As redes de números 1, 2 e 3 são predominantemente reclusas em suas universidades. As redes 2 e 3 possuem um ponto de convergência manifestado pela Universidade de São Paulo (USP). Neste item, buscou-se demonstrar o grau de centralidade dos autores, mas, como cada um representa uma determinada Instituição de Ensino Superior (IES), foi possível verificar que, mesmo com um alto grau de centralidade entre os atores na rede social de inovação, existe um pequeno movimento de cooperação entre as IES. Isso já foi evidenciado no estudo de Rossoni e Guarido Filho (2009), no qual os autores identificaram uma estrutural do tipo small worlds nos programas de pós-graduação em Administração. Ainda assim, mesmo que internamente, algumas universidades se destacam por seus grupos de pesquisa, como é o caso da Universidade Regional de Blumenau (FURB), Universidade Federal de Santa Maria (UFSM) e Faculdade de Economia, Administração e Contabilidade de Ribeirão Preto/USP.

\subsection{Centralidade de proximidade (closeness)}

A centralidade de proximidade é baseada na proximidade ou distância de um ator em relação aos outros atores em uma rede. A Tabela 3 apresenta os 15 primeiros atores com maior centralidade de proximidade, porém percebe-se que a média de proximidade permaneceu igual para todos, indicando que a distância geodésica entre esses atores na rede social de inovação é a mesma.

Tabela 3 - Centralidade de proximidade (closeness)

\begin{tabular}{c|c|c|c}
\hline Classificação & Principais atores & Farness & Closeness \\
\hline 1 & VACCARO G. L. R. & 450212.000 & 0.150 \\
\hline 2 & BITENCOURT M. P. & 450215.000 & 0.150 \\
\hline 3 & PELLEGRIN I. & 450216.000 & 0.150 \\
\hline 4 & LACERDA D. P. & 450216.000 & 0.150 \\
\hline 5 & ZIMMER M. V. & 450216.000 & 0.150 \\
\hline 6 & POHLMANN C. R. & 450218.000 & 0.150 \\
\hline 7 & CAMARGO L. F. R. & 450218.000 & 0.150 \\
\hline 8 & SILVA D. O. & 450218.000 & 0.150 \\
\hline 10 & ANTUNES JR. J. A. V. & 450223.000 & 0.150 \\
\hline 11 & LEIS R. P. & 450223.000 & 0.150 \\
\hline 12 & BATISTA G. D. M. & 450225.000 & 0.150 \\
\hline 13 & SILVA J. M. & 450225.000 & 0.150 \\
\hline 14 & SILVA C. E. S. & 450234.000 & 0.150 \\
\hline 15 & MARCANTONIO M. I. P. & 452238.000 & 0.150 \\
\hline FEREIRA R. S. & & 0.150 \\
\hline
\end{tabular}

Fonte: dados da pesquisa.

A medida de centralidade de proximidade (closeness) de um ator é obtida por meio da soma das distâncias geodésicas entre todos os outros atores (SCOTT, 2000; WASSERMAN; FAUST, 1994). Distância geodésica implica "[...] o caminho mais curto possível entre um ator e outro" (HANNEMAN; RIDDLE, 2005, p. 87). Isso significa que os atores VACCARO G. L. R., BITENCOURT M. P., PELLEGRIN I., LACERDA D. P., ZIMMER M. V., POHLMANN C. R., CAMARGO L. F. R., SILVA D. 
O., ANTUNES JR. J. A. V., LEIS R. P., BATISTA G. D. M., SILVA J. M., SILVA C. E. S., MARCANTONIO M. I. P. e PEREIRA R. S. são os atores que percorrem o menor percurso para alcançar os outros elos da rede. Essa pequena distância significa que a informação circula mais facilmente entre esses atores, possibilitando maior compartilhamento de informações. Os autores VACCARO G. L. R. e BITENCOURT M. P. também pertencem ao grupo que compõe a centralidade de intermediação.

\subsection{Centralidade de intermediação (betweenness)}

A centralidade de intermediação é baseada no quanto um ator facilita o fluxo de informação em determinada rede. A Tabela 4 apresenta os 11 primeiros atores com maior centralidade de intermediação. Esse dado se revela relativamente pequeno, dado o tamanho total da rede social de inovação, demonstrada na Figura 1. Porém esses atores são os conectores que favorecem o acesso a qualquer outro na rede a partir de seus relacionamentos; mesmo que a maioria não esteja relacionada diretamente entre si, eles se conectam indiretamente por meio desses poucos intermediários. Esse fenômeno também pode ser chamado de Small World ou "Mundo Pequeno" (MILGRAM, 1967; WATTS; STROGATZ, 1998, LAZZARINI, 2007).

Tabela 4 - Centralidade de intermediação (betweenness)

\begin{tabular}{c|c|c}
\hline Classificação & Nome dos principais atores & Betweenness \\
\hline 1 & VACCARO G. L. R. & 0,018 \\
\hline 2 & BITENCOURT M. P. & 0,013 \\
\hline 3 & GOMES C. M. & 0,011 \\
\hline 4 & PEREIRA R. S. & 0,010 \\
\hline 5 & MACHADO D. P. N. & 0,010 \\
\hline 6 & PORTO G. S. & 0,009 \\
\hline 7 & BRESCIANI L. P. & 0,007 \\
\hline 8 & VARGAS E. R. & 0,006 \\
\hline 9 & GUIMARÃES T. A. & 0,004 \\
\hline 10 & KNEIPP J. M. & 0,004 \\
\hline 11 & GODOY L. P. & 0,004 \\
\hline
\end{tabular}

Fonte: dados da pesquisa.

A Tabela 4 demonstra que os atores VACCARO G. L. R., BITENCOURT M. P., PORTO G. S., GOMES C. M., PEREIRA R. S., MACHADO D. P. N., PORTO G. S., BRESCIANI L. P., VARGAS E. R., GUIMARÃES T. A., KNEIPP J. M. e GODOY L. P. são os autores intermediários que mais conectam autores. Também é possível verificar a relevância dos autores VACCARO G. L. R. e BITENCOURT M. P., que são os principais conectores de grupos distintos, favorecendo a expansão da rede social de inovação. 


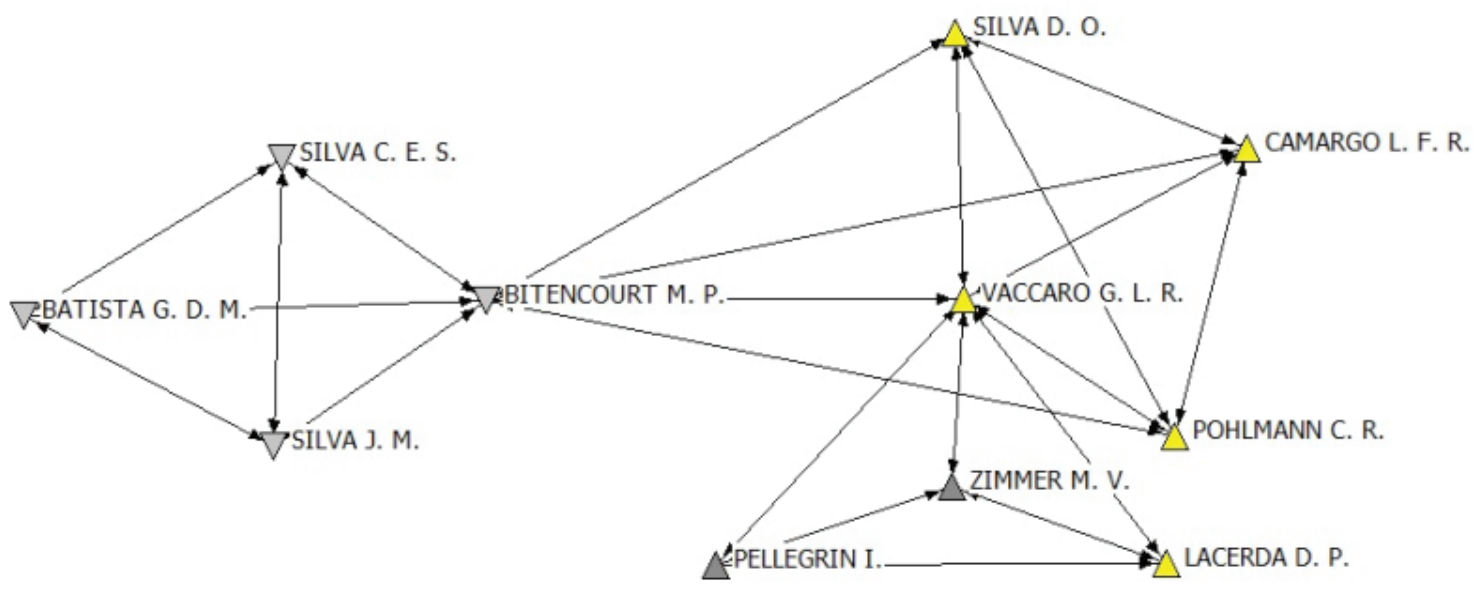

Legenda:

\begin{tabular}{|c|c|}
\hline$\nabla$ & Unifei \\
\hline$\Delta$ & Unisinos \\
\hline$\triangle$ & Produttare Consultores Associados \\
\hline
\end{tabular}

Fonte: dados da pesquisa.

A centralidade de intermediação pode ser considerada um dos indicadores mais importantes de uma rede social, pois, por meio dela, um ator interage entre atores não adjacentes (SCOTT, 2000; WASSERMAN; FAUST, 1994). Um ator é um intermediário quando se liga a vários outros atores que não se conectam a ele diretamente.

A Figura 3 demonstra a intermediação de dois autores - VACCARO G. L. R. e BITENCOURT M. P. - que, juntos, conectam uma rede formada por outros nove pesquisadores, a maior rede de intermediação possível. A rede do ator VACCARO G. L. R. é composta, quase que em sua totalidade, por atores da Unisinos; já a rede de BITENCOURT M. P. estabelece seu número de parcerias exclusivamente com a Unifei.

É importante ressaltar que são os autores, e não as instituições, que formam as redes, porém estar inserido em uma organização que favorece a construção do conhecimento pode incentivar relações interinstitucionais. Quanto mais laços forem criados, maior a possibilidade de se construir uma rede de intermediação densa.

Essa intermediação resulta em interação essencial para geração de inovação e transpassa os muros da academia, pois organizações também precisam de fontes de informações e conhecimentos que podem estar dentro ou fora da empresa. Como a organização não inova sozinha, essa interação com vários agentes (econômicos e sociais) facilita o seu processo de inovação (LEMOS; LASTRES; ALBAGLI, 1999). Neste estudo, observou-se, mesmo que de forma tímida, que em cerca de $2 \%$ dos artigos ocorreu essa interação. No levantamento das instituições pertencentes a cada autor que teve seus artigos publicados nos periódicos analisados, em alguns casos foi possível perceber a conjunção entre a IES e empresas privadas, tais como a Produttare Consultores Associados, Senac, Embrapa, entre outras. 


\subsection{Análise dos clusters}

Dos 678 atores que formaram o campo de produção científica em inovação, foram encontrados 133 cliques ou clusters de tamanho até três autores (WASSERMAN; FAUST, 1994; SCOTT, 2000). Os demais clusters foram compostos por 64 subgrupos com quatro autores, 39 subgrupos com cinco autores, 26 subgrupos com seis autores, 13 subgrupos com sete autores e, por fim, oito subgrupos com oito autores, sendo este último o principal grupo, identificado no Quadro 1.

Quadro $1-N$-cliques

\begin{tabular}{|l|}
\hline N-ClIQUES \\
\hline Max Distance (n-): 2 \\
\hline Minimum Set Size: 8 \\
\hline Atores: 678 \\
\hline 133-cliques found. \\
\hline SILVA J. M.; SILVA C. E. S.; BATISTA G. D. M.; BITENCOURT M. P.; VACCARO G. L. R.; SILVA D. O.; CAMARGO \\
L. F. R.; POHLMANN C. R. \\
\hline BITENCOURT M. P.; VACCARO G. L. R.; SILVA D. O.; CAMARGO L. F. R.; POHLMANN C. R.; PELLEGRIN I.; \\
ZIMMER M. V.; LACERDA D. P. \\
\hline VENDEMIATTI M.; SIQUEIRA E. S.; FILARDI F.; BINOTTO E.; SIMIONI F. J.; HOFF D. N.; NAKAYAMA M. K.; \\
CASAROTTO E. L. \\
\hline GOMES C. M.; KRUGLIANSKAS I.; SCHERER F. L.; MENEZES U. G.; KNEIPP J. M.; BARBIERI L. A.; WITTMANN \\
M. L.; LÜBECK R. M. \\
\hline GOMES C. M.; KRUGLIANSKAS I.; SCHERER F. L.; MENEZES U. G.; KNEIPP J. M.; BARBIERI L. A.; CARPES A. \\
M.; BEURON T. A. \\
\hline PACAGNELLA JR. A. C.; PORTO G. S.; SILVA S. L.; KANNEBLEY JR. S.; SALGADO JR. A. P.; FIGLIOLI A.; DIAS A. \\
A.; JOÃO I. S.; GALINA S. V. R. \\
\hline MACHADO D. P. N.; CARVALHO L. C.; HEINZMANN L. M.; BARZOTTO L. C.; GOMES G.; GIOTTO O. T.; SCAR- \\
PIN M. R. S.; MONDINI L. C.; NEUMANN M. \\
\hline PEREIRA R. S.; FRANCO I. D.; ALMEIDA L. C. B.; SANTOS I. C.; OLIVEIRA S. M.; HAYASHI JR. P.; ARRUDA A. \\
G.; BRESCIANI L. P.; SILVA T. N. \\
\hline
\end{tabular}
Fonte: dados da pesquisa.

Em qualquer rede social, alguns elos mantêm as relações mais estreitas. Essas relações formam subgrupos, que são baseados na alcançabilidade e na proximidade de indivíduos e que são formalizados pelo conceito $n$-clique (WASSERMAN; FAUST, 1994; SCOTT, 2000). Neste estudo, é possível observar que, mesmo que essa rede se apresente fragmentada, a existência de 133 subgrupos indica que os estudiosos na área de inovação produzem em rede, mesmo sendo ela restrita a seus pares.

O que se percebe nesta análise é a necessidade de expansão da rede social de inovação, pois, como apresentado no referencial teórico, a inovação necessita de conexões e, quanto mais conectada uma rede estiver, maior o fluxo de informação circulando por ela, favorecendo a qualidade das informações apresentadas nos artigos publicados. Conforme Monteiro, Sacomano Neto e Giuliani (2012), as redes de inovação, ao se tornarem um mecanismo para difundir a novidade por meio de empresas e pessoas, colaborando e interagindo entre si, surgem como uma nova forma de organização para gerar conhecimento.

Porém é importante lembrar que somente os laços efetivamente construídos não consolidam uma rede. É necessário que esses laços tenham continuidade e diversidade na produção científica, pois, se o número de laços não acompanhar o número possível na rede, poderá ocorrer uma maior fragmentação da rede (MARTINS, 2009). 


\section{CONCLUSÕES E RECOMENDAÇÕES}

Neste estudo, procurou-se evidenciar quais atores formam as redes sociais a partir da produção científica sobre inovação dos periódicos de alto impacto, brasileiros, listados na Capes, classificados como Qualis A1, A2, B1 e B2.

$\mathrm{Na}$ análise de redes sociais de inovação, foram encontrados 678 autores, que representam os "nós" que, juntos, produziram 1.607 relações entre si - chamadas de "laços" - de 459.006 laços possíveis, indicando uma rede social formada por laços fracos, fragmentada, com a existência de 133 subgrupos e de baixa densidade. Esse resultado corrobora estudos como o de Martins (2009) e Guimarães et al. (2009). O primeiro identificou nas publicações do campo de gestão de operações um déficit entre o volume de laços construídos entre os autores e o aumento de laços possíveis, levando a uma maior fragmentação da rede. Já Guimarães et al. (2009) também identificaram uma rede pouco densa, com relações esparsas e, na maioria das vezes, fracas entre pesquisadores de programas de pós-graduação.

Por uma questão de delimitação do estudo, optou-se por analisar as medidas de densidade, grau de centralidade, centralidade de proximidade, centralidade de intermediação e análise dos clusters. No caso do grau de centralidade, verificou-se um conjunto de atores que compartilham conhecimento e desenvolvem trabalhos em equipe, porém restritos ao seu grupo, limitando o conhecimento entre os seus pares. Contudo foi possível observar que, mesmo com alto grau de centralidade entre os atores na rede social de inovação, existe uma cooperação entre as IES. Essa tendência à centralidade reforça os achados de Mendes-da-Silva, Onusic e Giglio (2013), que identificaram na produção brasileira uma formação de pesquisadores com maior grau de centralidade.

A centralidade de proximidade permaneceu igual para os principais atores da rede, indicando que a informação circula mais facilmente entre esses atores, o que possibilita maior fluxo de informações. Alguns dos autores, como VACCARO G. L. R., BITENCOURT M. P., PELLEGRIN I., LACERDA D. P. e ZIMMER M. V., também pertencem ao grupo que compõe a centralidade de intermediação.

Os atores intermediários são os mais importantes em uma rede social, pois, por meio deles, um ator interage entre atores não adjacentes. Neste estudo, destacam-se os autores VACCARO G. L. R. e BITENCOURT M. P., pois eles são os principais conectores entre dois grupos de redes sociais distintas, que, juntos, conectam uma rede formada por outros nove pesquisadores, a maior rede de intermediação possível. Essa rede favorece a expansão da rede social de inovação, pois autores que participam da rede de centralidade de intermediação compartilham várias linhas de pensamento diferentes, estendendo seu conhecimento para além de um restrito conjunto de pesquisadores, gerando ideias sobre novos temas a serem pesquisados ou contribuindo para estudos em andamento. Com isso, é possível afirmar que o fluxo de informações que circulam na rede social pode gerar inovações na produção científica e beneficiar toda uma sociedade.

A centralidade de intermediação ou estrutura do tipo small world (MILGRAM, 1967; WATTS; STROGATZ, 1998, LAZZARINI, 2007) foi também evidenciada nos estudos de Rossoni e Guarido Filho (2009) em relação aos programas de pós-graduação em Administração. Da mesma forma, Mendes-da-Silva, Onusic e Giglio (2013) caracterizaram o ambiente brasileiro como formado por small worlds e com baixa regularidade de produção.

O estudo demonstrou que a rede social de inovação é composta por 133 subgrupos, que trabalham conectados com, no mínimo, três autores, mas que restringem sua produção a seus pares, evidenciando uma necessidade de expansão da rede social de inovação, não somente em laços constituídos, mas também em continuidade e diversidade entre autores e, consequentemente, entre IES. Uma rede mais densa, em princípio, significa difusão mais rápida de informações, pois o conhecimento chega com maior agilidade e fluidez àqueles conectados a ela, contribuindo para a ampliação, a variação e as inovações do capital intelectual. Uma rede pouco densa também foi identificada por Martins et al. (2010), ao analisarem a rede de pesquisadores da área de gestão de operações no Brasil. 
Espera-se que esta pesquisa contribua para o campo teórico sobre redes sociais, bem como alerte os pesquisadores de instituições de ensino privadas e governamentais sobre a importância de compartilhar o conhecimento. A inovação é um processo de aprendizagem interativo, contínuo e heterogêneo, que ocorre com a contribuição de vários agentes, no qual cada qual um traz consigo seu conhecimento e sua lente sobre determinado assunto. Como relatado por Popper (1972) e Kuhn (1978), o conhecimento científico deve ser construído socialmente. Para futuros estudos, sugere-se estender a pesquisa também para a área de eventos, bem como fazer um levantamento das redes sociais de inovação em periódicos internacionais.

\section{REFERENCIAS}

AFUAH, A. Innovation management: strategies, implementation and profits. New York: Oxford University Press, 2003.

ARBIX, G.; DE NEGRI, J. A. A Inovação no centro da Agenda do desenvolvimento. Brasil Pós-Crise: agenda para a próxima década. 2. ed. Rio de Janeiro: Campus-Elsevier, 2009.

BARABÁSI, A. Network theory-the emergence of the creative enterprise. Science, v. 308, n. 5.722, p. 639-641, 2005.

BARBIERI, J. C. A contribuição da área produtiva no processo de inovação tecnologias. RAE - Revista de Administração de Empresas, v. 37, n. 1, p. 66-77, 1997.

BART, C. Innovation, mission statements and learning. International Journal of Technology Management, v. 27, n. 6, p. 544-561, 2004.

BERKOWITZ, S. D. An introduction to structural analysis: the network approach to social research. Toronto: Butterworth-Heinemann, 1982.

BIGNETTI, L. P. Gestão de tecnologia e inovação: uma análise de autores, vertentes teóricas e estratégias metodológicas predominantes em trabalhos apresentados nos encontros da ANPAD. In: ENCONTRO ANUAL DA ANPAD, 30., 2006, Salvador. Anais... Salvador: ANPAD, 2006.

BIGNETTI, L. P.; CAPPRA, C. M.; THOMAS, E. Estudos nacionais e internacionais sobre gestão da inovação: uma análise dos principais autores e das vertentes teóricas atuais. In: ENCONTRO ANUAL DA ANPAD, 32., 2008, Rio de Janeiro. Anais... Rio de Janeiro: ANPAD, 2008.

BULGACOV, S.; VERDU, F. C. Redes de pesquisadores da área de administração: um estudo exploratório. Revista de Administração Contemporânea, v. 5, n. SPE, p. 163-182, 2001.

BURT, R. S. Structural holes: the social structures of competition. Cambridge: Harvard University Press, 1992.

Coordenação de Aperfeiçoamento de Pessoal de Nível Superior (CAPES). Classificação de periódicos. 2008. Disponível em: <http:// http://www.capes.gov.br/>. Acesso em: 13 maio 2008.

. Classificação de periódicos. 2011. Disponível em: <http:// http://www.capes.gov.br/>. Acesso em: 21 junho 2011.

CERVO, A. L.; BERVIAN, A. Metodologia científica: para uso dos estudantes universitários. 3. ed. São Paulo: McGraw-Hill, 1983. 
CONCEIÇÃO, O. A. C. A centralidade do conceito de inovação tecnológica no processo de mudança estrutural. Ensaios FEE, v. 21, n. 2, p. 58-76, 2000.

DAMANPOUR, F. Organizational innovation: a meta-analysis of effects of determinants and moderators. Academy of Management Journal, v. 34, n. 3, p. 555-590, 1991.

DE ANDRADE JR., P. P.; CERANTO, F. A. A. Um retrato bibliométrico da produção científica brasileira sobre Gestão da Tecnologia e da Inovação no período 2001-2011. Revista de Administração da UFSM, v. 6, n. 4, p. 708-719, 2013.

DOSI, G. Technological paradigms and technological trajectories: a suggested interpretation of the determinants and directions of technical change. Research Policy, v. 11, n. 3, p. 147-162, 1982.

. Sources, procedures and microeconomic effects of innovation. Journal of Economic Literature, v. 26, n. 3, p. 1.120-1.171, 1988.

GALASKIEWICZ, J.; WASSERMAN, S. (Ed.). Advances in social network analysis: research in the social and behavioral sciences. London: Sage, 1994.

GARDNER, J. W. A liderança nas cidades. In: Hesselbein, F.; Cohen, P. M. (Org.). De líder para líder. São Paulo: Futura, 1999. p. 361-367.

GNYAWALI, D. R.; MADHAVAN, R. Cooperative networks and competitive dynamics: a structural embeddedness perspective. Academy of Management Review, v. 26, n. 3, p. 431-445, 2001.

GOMES, G.; MACHADO, D. D. P. N.; GIOTTO, O. T. Análise do conteúdo dos artigos de inovação publicados nos anais do ALTEC, SIMPOI e EnANPAD (2003-2007). In: SIMPÓSIO DE ADMINISTRAÇÃO DA PRODUÇÃO, LOGÍSTICA E OPERAÇÕES INTERNACIONAIS-SIMPOI, 2009, São Paulo. Anais... São Paulo: SIMPOI, 2009.

GUIMARÃES, T. A. et al. A rede de programas de pós-graduação em administração no Brasil: análise de relações acadêmicas e atributos de programas. Revista de Administração Contemporânea, v. 13, n. 4, p. 564-582, 2009.

GUIMERÀ, R. et al. Team assembly mechanisms determine collaboration network structure and team performance. Science, v. 308, n. 5.722, p. 697-702, 2005.

GUNDLING, E. The 3M Way to innovation: balancing people and profit. New York: Vintage Books, 1999.

HAMEL, G. O laboratório de inovação em gestão. HSM Management, v. 58, set./out. 2006.

HANNEMAN, R. A.; RIDDLE, M. Introduction to social network methods. Riverside: University of California, 2005.

KNEIPP, J. M. et al. Emergencia temática da inovação sustentável: uma análise da produção científica através da base web of science. Revista de Administração da UFSM, v. 4, n. 3, p. 442-457, 2011.

KNOKE, D.; KUKLINSKI, J. H. (Ed.). Network analysis. Newsbury Sage, 1982.

KUHN, T. S. A estrutura das revoluções científicas. 2. ed. São Paulo: Perspectiva, 1978.

LARIVIÈRE, V.; GINGRAS, Y.; ARCHAMBAULT, E. Comparative analysis of networks of collaboration of Canadian researchers in the natural sciences, social sciences and the humanities. Scientometrics, v. 68, n. 3, p. 519-533, 2006. 
LEMOS, C.; LASTRES, H. M. M.; ALBAGLI, S. Inovação na era do conhecimento. In: ASTRES, H. M. M.; ALBAGLI, S. (Org.). Informação e globalização na era do conhecimento. Rio de Janeiro: Campus, 1999. p. 122-144.

LAZZARINI, S. G. Mudar tudo para não mudar nada: análise da dinâmica de redes de proprietários no Brasil como mundos pequenos. RAE-eletrônica, v. 6, n. 1, 2007.

LIU, X. et al. Co-authorship networks in the digital library research community. Information Processing \& Management, v. 41, n. 6, p. 1462-1480, 2005.

MACHADO, D. D. P. N.; GOMES, G.; GIOTTO, O. O que se produz de conhecimento sobre inovação: uma breve análise das características dos artigos de inovação publicados nos anais do ENANPAD (19972007). In: SIMPÓSIO DE ADMINISTRAÇÃO DA PRODUÇÃO, LOGÍSTICA E OPERAÇÕES INTERNACIONAIS - SIMPOI, 2008, São Paulo. Anais... São Paulo: SIMPOI, 2008.

MACIAS-CHAPULA, C. A. O papel da informetria e da cienciometria e sua perspectiva nacional e internacional. Ciência da Informação, v. 27, n. 2, p.64-68, 1998.

MARTINS, G. A.; THEÓPHILO, C. R. Metodologia da investigação científica para ciências sociais aplicadas. São Paulo: Atlas, 2007.

MARTINS, G. S. A construção do conhecimento científico no campo de gestão de operações no Brasil: uma análise sob a ótica de redes sociais do período 1997-2008. 2009. 179 p. Dissertação (Mestrado em Administração)-Fundação Getúlio Vargas, São Paulo, 2009.

. et al. Gestão de operações no Brasil: uma análise do campo científico a partir da rede social de pesquisadores. RAE-eletrônica, v. 9, n. 2, 2010.

MENDES-DA-SILVA, W.; ONUSIC, L. M.; GIGLIO, E. M. The finance research network in Brazil: a small world. Revista de Administração Contemporânea, v. 17, n. 6, p. 739-763, 2013.

MILGRAM, S. The small world problem. Psychology Today, v. 2, n. 1, p. 60-67, 1967.

MOODY, J. The structure of a social science collaboration network: disciplinary cohesion from 1963 to 1999. American Sociological Review, v. 69, n. 2, p. 213-238, 2004.

MONTEIRO, T. A.; SACOMANO NETO, M.; GIULIANI, A. C. Redes de inovação e composto mercadológico: um estudo exploratório sobre o desenvolvimento de produtos. Revista de Administração da UFSM, v. 6 , n. 4, p. 672-684, 2013.

MUYLDER, C. F. et al. Tema inovação: uma análise bibliométrica no Evento da EnANPAD 2007. Gestão \& Tecnologia, v. 8, n. 1, p. 1-13, jan./jul. 2008.

NELSON, R. Uso da análise de redes sociais no estudo das estruturas organizacionais. Revista de Administração de Empresas, v. 24, n. 4, p. 150-157, out./dez. 1984.

NEWMAN, M. E. J. Scientific collaboration networks. I. Network construction and fundamental results. Physical review E, v. 64, n. 1, p. 1-8, 2001a.

. The structure of scientific collaboration networks. Proceedings of the National Academy of Sciences, v. 98, n. 2, p. 404-409, 2001b.

NOHRIA, N. Introduction: Is a network perspective a useful way of studying organizations. Networks and Organizations: Structure, Form, and Action, v. 1, p. 22, 1992. 
OTTE, E.; ROUSSEAU, R. Social network analysis: a powerful strategy, also for the information sciences. Journal of information Science, v. 28, n. 6, p. 441-453, 2002.

Pennings, J. M. Innovations as precursors of organizational performance. In: Galliers, R. D.; Baets, W. R. J. (Ed.). Information technology and organizational transformation: innovation for the 21st century organization. New York: John Wiley \& Sons, 1998.

PEREIRA, J. A.; REINERT, M. A influência das redes sociais na inovação: um estudo de caso em uma incubada do Centro Incubador Tecnológico-CIT/FUNDETEC. Revista Eletrônica de Ciência Administrativa - RECADM, v. 12, n. 2, p. 140-155, 2013.

PODOLNY, J. M.; PAGE, K. L. Network forms of organization. Annual Review of Sociology, v. 24, n. 1, p. 57-76, 1998.

POPPER, K. R. Conjecturas e refutações. Brasília: UNB, 1972.

PRICE, D. J. S. Networks of scientific papers. Science, v. 149, p. 510-515, 1965.

RAPOPORT, A.; HORVATH, W. J. A study of a large sociogram. Behavioral Science, v. 6, n. 4, p. 279-291, 1961.

REDNER, S. How popular is your paper? An empirical study of the citation distribution. The European Physical Journal B-Condensed Matter and Complex Systems, v. 4, n. 2, p. 131-134, 1998.

ROPELATO, M., SILVEIRA, A.; MACHADO, D. D. P. N. Inovaç̧ão: análise da produção cientifica brasileira:20062009. In: SIMPÓSIO DE ADMINISTRAÇÃO DA PRODUÇÃO, LOGÍSTICA E OPERAÇÕES INTERNACIONAIS SIMPOI, 2010, São Paulo. Anais... São Paulo: SIMPOI, 2010.

ROSSONI, L.; HOCAYEN-DA-SILVA, A. J.; FERREIRA JR., I. Aspectos estruturais da cooperação entre pesquisadores no campo de administração pública e gestão social: análise das redes entre instituições no Brasil. Revista de Administração Pública, v. 42, n. 6, p. 1.041-1.067, 2009.

ROSSONI, L.; GUARIDO FILHO, E. R. Cooperação entre programas de pós-graduação em Administração no Brasil: evidências estruturais em quatro áreas temáticas. Revista de Administração Contemporânea, v. 13, p. 366-390, 2009.

ROTHWELL, R. Towards the fifth-generation innovation process. International Marketing Review, v. 11, n. 1, p. 7-31, 1994.

SCHUMPETER, J. Theorie der wirtschaftlichen Entwicklung. Leipzig: Duncker und Humblot, 1911.

. Teoria do desenvolvimento econômico: uma investigação sobre lucros, capital, crédito, juro e o ciclo econômico. São Paulo: Abril Cultural, 1982.

SCOTT, J. Social Network Analysis: a handbook. 2. ed. London: Sage Publications, 2000.

SILVA, A. B. O. et al. Análise de redes sociais como metodologia de apoio para a discussão da interdisciplinaridade na ciência da informação. Ciência da Informação, v. 35, n. 1, p. 72-93, 2006.

TIDD, J.; BESSANT, J.; PAVITT, K. Managing innovation: integrating technological, managerial organizational change. New York: John Wiley \& Sons, 2005.

TOMAÉL, M. I.; ALCARÁ, A. R.; DI CHIARA, I. G. Das redes sociais à inovação. Ciência da Informação, Brasília, v. 34, n. 2, p. 93-104, 2005. 
UZZI, B. Social structure and competition in interfirm networks: the paradox of embeddedness. Administrative Science Quarterly, p. 35-67, 1997.

VAN DE VEN, A. H.; ANGLE, H. L. An introduction to the Minnesota innovation research program. In: .; POOLE, M. S. (Org.). Research on the management of innovation. New York: Ballinger/Harper \& Row, 1989. p. 3-30.

VAN DE VEN, A. H.; POOLE, M. S. Methods for studying innovation development in the Minnesota Innovation Research Program. Organization Science, v. 1, n. 3, p. 313-335, 1990.

VERGARA, S. C. Projetos e relatórios de pesquisa em administração. 3. ed. São Paulo: Atlas, 2000.

WAN, D.; ONG, C. H.; LEE, F. Determinants of firm innovation in Singapore. Technovation, v. 25, n. 3, p. 261-268, 2005.

WASSERMAN, S; FAUST, K. Social network analysis: methods and applications. New York: Cambridge University Press, 1994.

WATTS, D. J.; STROGATZ, S. H. Collective dynamics of 'small-world'networks. Nature, v. 393, n. 6.684, p. 440-442, 1998.

ZALTMAN, G.; DUNCAN, R.; HOLBEK, J. Innovations and organizations. New York: Wiley, 1973. 